\title{
Training v-Support Vector Regression: Theory and Algorithms
}

\author{
Chih-Chung Chang \\ b4506055@csie.ntu.edu.tw \\ Chih-Jen Lin \\ cjlin@csie.ntu.edu.tw \\ Department of Computer Science and Information Engineering, National Taiwan \\ University, Taipei 106, Taiwan
}

We discuss the relation between $\epsilon$-support vector regression $(\epsilon$-SVR) and $v$-support vector regression ( $v$-SVR). In particular, we focus on properties that are different from those of C-support vector classification (C-SVC) and $v$-support vector classification ( $v$-SVC). We then discuss some issues that do not occur in the case of classification: the possible range of $\epsilon$ and the scaling of target values. A practical decomposition method for $v$-SVR is implemented, and computational experiments are conducted. We show some interesting numerical observations specific to regression.

1 Introduction

The v-support vector machine (Schölkopf, Smola, Williamson, \& Bartlett, 2000; Schölkopf, Smola, \& Williamson, 1999) is a new class of support vector machines (SVM). It can handle both classification and regression. Properties on training $v$-support vector classifiers ( $v$-SVC) have been discussed in Chang \& Lin (2001b). In this letter, we focus on $v$-support vector regression $\left(\nu\right.$-SVR). Given a set of data points, $\left\{\left(\mathbf{x}_{1}, y_{1}\right), \ldots,\left(\mathbf{x}_{l}, y_{l}\right)\right\}$, such that $\mathbf{x}_{i} \in R^{n}$ is an input and $y_{i} \in R^{1}$ is a target output, the primary problem of $v$-SVR is as follows:

$$
\begin{gathered}
\min \frac{1}{2} \mathbf{w}^{T} \mathbf{w}+C\left(v \epsilon+\frac{1}{l} \sum_{i=1}^{l}\left(\xi_{i}+\xi_{i}^{*}\right)\right) \\
\left(\mathbf{w}^{T} \phi\left(\mathbf{x}_{i}\right)+b\right)-y_{i} \leq \epsilon+\xi_{i}, \\
y_{i}-\left(\mathbf{w}^{T} \phi\left(\mathbf{x}_{i}\right)+b\right) \leq \epsilon+\xi_{i}^{*}, \\
\xi_{i}, \xi_{i}^{*} \geq 0, i=1, \ldots, l, \epsilon \geq 0 .
\end{gathered}
$$

Here, $0 \leq v \leq 1, C$ is the regularization parameter, and training vectors $\mathbf{x}_{i}$ are mapped into a higher (maybe infinite) dimensional space by the function $\phi$. The $\epsilon$-insensitive loss function means that if $\mathbf{w}^{T} \phi(\mathbf{x})$ is in the range of $y \pm \epsilon$, 
no loss is considered. This formulation is different from the original $\epsilon$-SVR (Vapnik, 1998):

$$
\begin{aligned}
\min & \frac{1}{2} \mathbf{w}^{T} \mathbf{w}+\frac{C}{l} \sum_{i=1}^{l}\left(\xi_{i}+\xi_{i}^{*}\right) \\
& \left(\mathbf{w}^{T} \phi\left(\mathbf{x}_{i}\right)+b\right)-y_{i} \leq \epsilon+\xi_{i}, \\
& y_{i}-\left(\mathbf{w}^{T} \phi\left(\mathbf{x}_{i}\right)+b\right) \leq \epsilon+\xi_{i}^{*}, \\
& \xi_{i}, \xi_{i}^{*} \geq 0, i=1, \ldots, l .
\end{aligned}
$$

As it is difficult to select an appropriate $\epsilon$, Schölkopf et al. (1999) introduced a new parameter $v$ that lets one control the number of support vectors and training errors. To be more precise, they proved that $v$ is an upper bound on the fraction of margin errors and a lower bound of the fraction of support vectors. In addition, with probability 1, asymptotically, $v$ equals both fractions.

Then there are two different dual formulations for $P_{\nu}$ and $P_{\epsilon}$ :

$$
\begin{aligned}
\min & \frac{1}{2}\left(\boldsymbol{\alpha}-\boldsymbol{\alpha}^{*}\right)^{T} \mathbf{Q}\left(\boldsymbol{\alpha}-\boldsymbol{\alpha}^{*}\right)+\mathbf{y}^{T}\left(\boldsymbol{\alpha}-\boldsymbol{\alpha}^{*}\right) \\
& \mathbf{e}^{T}\left(\boldsymbol{\alpha}-\boldsymbol{\alpha}^{*}\right)=0, \mathbf{e}^{T}\left(\boldsymbol{\alpha}+\boldsymbol{\alpha}^{*}\right) \leq C \nu, \\
& 0 \leq \alpha_{i}, \alpha_{i}^{*} \leq C / l, \quad i=1, \ldots, l . \\
\min & \frac{1}{2}\left(\boldsymbol{\alpha}-\boldsymbol{\alpha}^{*}\right)^{T} \mathbf{Q}\left(\boldsymbol{\alpha}-\boldsymbol{\alpha}^{*}\right)+\mathbf{y}^{T}\left(\boldsymbol{\alpha}-\boldsymbol{\alpha}^{*}\right)+\epsilon \mathbf{e}^{T}\left(\boldsymbol{\alpha}+\boldsymbol{\alpha}^{*}\right) \\
& \mathbf{e}^{T}\left(\boldsymbol{\alpha}-\boldsymbol{\alpha}^{*}\right)=0, \quad i=1, \ldots, l, \\
& 0 \leq \alpha_{i}, \alpha_{i}^{*} \leq C / l, \quad i=1,
\end{aligned}
$$

where $Q_{i j} \equiv \phi\left(\mathbf{x}_{i}\right)^{T} \phi\left(\mathbf{x}_{j}\right)$ is the kernel and $\mathbf{e}$ is the vector of all ones. Then the approximating function is

$$
f(\mathbf{x})=\sum_{i=1}^{l}\left(\alpha_{i}^{*}-\alpha_{i}\right) \phi\left(\mathbf{x}_{i}\right)^{T} \phi(\mathbf{x})+b .
$$

For regression, the parameter $v$ replaces $\epsilon$ while in the case of classification, $v$ replaces $C$. In Chang \& Lin (2001b), we discussed the relation between $\nu$-SVC and C-SVC as well as how to solve $v$-SVC in detail. Here, we are interested in different properties for regression. For example, the relation between $\nu$-SVR and $\epsilon$-SVR is not the same as that between $\nu$-SVC and C-SVC. In addition, similar to the situation of C-SVC, we make sure that the inequality $\mathbf{e}^{T}\left(\boldsymbol{\alpha}+\boldsymbol{\alpha}^{*}\right) \leq C \nu$ can be replaced by an equality so algorithms for $v$-SVC can be applied to $v$-SVR. They will be the main topics of sections 2 and 3 .

In section 4 , we discuss the possible range of $\epsilon$ and show that it might be easier to use $v$-SVM. We also demonstrate some situations where the 
scaling of the target values $\mathbf{y}$ is needed. Note that these issues do not occur for classification. Finally, section 5 presents computational experiments. We discuss some interesting numerical observations that are specific to support vector regression.

\section{The Relation Between $\nu$-SVR and $\epsilon$-SVR}

In this section, we will derive a relationship between the solution set of $\epsilon$-SVR and $\nu$-SVR that allows us to conclude that the inequality constraint, equation 1.3, can be replaced by an equality.

In the dual formulations mentioned earlier, $\mathbf{e}^{T}\left(\boldsymbol{\alpha}+\boldsymbol{\alpha}^{*}\right)$ is related to $C \nu$. Similar to Chang and Lin (2001b), we scale them to the following formulations so $\mathbf{e}^{T}\left(\boldsymbol{\alpha}+\boldsymbol{\alpha}^{*}\right)$ is related to $v$ :

$$
\begin{gathered}
\min \frac{1}{2}\left(\boldsymbol{\alpha}-\boldsymbol{\alpha}^{*}\right)^{T} \mathbf{Q}\left(\boldsymbol{\alpha}-\boldsymbol{\alpha}^{*}\right)+(\mathbf{y} / C)^{T}\left(\boldsymbol{\alpha}-\boldsymbol{\alpha}^{*}\right) \\
\mathbf{e}^{T}\left(\boldsymbol{\alpha}-\boldsymbol{\alpha}^{*}\right)=0, \mathbf{e}^{T}\left(\boldsymbol{\alpha}+\boldsymbol{\alpha}^{*}\right) \leq v \\
0 \leq \alpha_{i}, \alpha_{i}^{*} \leq 1 / l, \quad i=1, \ldots, l \\
\left(D_{\epsilon}\right) \quad \min \frac{1}{2}\left(\boldsymbol{\alpha}-\boldsymbol{\alpha}^{*}\right)^{T} \mathbf{Q}\left(\boldsymbol{\alpha}-\boldsymbol{\alpha}^{*}\right)+(\mathbf{y} / C)^{T}\left(\boldsymbol{\alpha}-\boldsymbol{\alpha}^{*}\right)+(\epsilon / C) \mathbf{e}^{T}\left(\boldsymbol{\alpha}+\boldsymbol{\alpha}^{*}\right) \\
\mathbf{e}^{T}\left(\boldsymbol{\alpha}-\boldsymbol{\alpha}^{*}\right)=0, \\
0 \leq \alpha_{i}, \alpha_{i}^{*} \leq 1 / l, \quad i=1, \ldots, l .
\end{gathered}
$$

For convenience, following Schölkopf et al. (2000), we represent $\left[\begin{array}{c}\boldsymbol{\alpha} \\ \boldsymbol{\alpha}^{*}\end{array}\right]$ as $\boldsymbol{\alpha}^{(*)}$.

Remember that for $v$-SVC, not all $0 \leq v \leq 1$ lead to meaningful problems of $\left(D_{v}\right)$. Here, the situation is similar, so in the following, we define a $v^{*}$, which will be the upper bound of the interesting interval of $v$.

Definition 1. Define $v^{*} \equiv \min _{\boldsymbol{\alpha}^{(*)}} \mathbf{e}^{T}\left(\boldsymbol{\alpha}+\boldsymbol{\alpha}^{*}\right)$, where $\boldsymbol{\alpha}^{(*)}$ is any optimal solution of $\left(D_{\epsilon}\right), \epsilon=0$.

Note that $0 \leq \alpha_{i}, \alpha_{i}^{*} \leq 1 / l$ implies that the optimal solution set of $D_{\epsilon}$ or $D_{v}$ is bounded. Because their objective and constraint functions are all continuous, any limit point of a sequence in the optimal solution set is in it as well. Hence, we have that the optimal solution set of $D_{\epsilon}$ or $D_{\nu}$ is close and bounded (i.e., compact). Using this property, if $\epsilon=0$, there is at least one optimal solution that satisfies $\mathbf{e}^{T}\left(\boldsymbol{\alpha}+\boldsymbol{\alpha}^{*}\right)=v^{*}$.

The following lemma shows that for $D_{\epsilon}, \epsilon>0$, at an optimal solution one of $\alpha_{i}$ and $\alpha_{i}^{*}$ must be zero:

Lemma 1. If $\epsilon>0$, all optimal solutions of $D_{\epsilon}$ satisfy $\alpha_{i} \alpha_{i}^{*}=0$. 
Proof. If the result is wrong, then we can reduce values of two nonzero $\alpha_{i}$ and $\alpha_{i}^{*}$ such that $\boldsymbol{\alpha}-\boldsymbol{\alpha}^{*}$ is still the same, but the term $\mathbf{e}^{T}\left(\boldsymbol{\alpha}+\boldsymbol{\alpha}^{*}\right)$ of the objective function is decreased. Hence, $\boldsymbol{\alpha}^{(*)}$ is not an optimal solution so there is a contradiction.

The following lemma is similar to Chang and Lin (2001b, lemma 4):

Lemma 2. If $\boldsymbol{\alpha}_{1}^{(*)}$ is any optimal solution of $D_{\epsilon_{1}}, \boldsymbol{\alpha}_{2}^{(*)}$ is any optimal solution of $D_{\epsilon_{2}}$, and $0 \leq \epsilon_{1}<\epsilon_{2}$, then

$$
\mathbf{e}^{T}\left(\boldsymbol{\alpha}_{1}+\boldsymbol{\alpha}_{1}^{*}\right) \geq \mathbf{e}^{T}\left(\boldsymbol{\alpha}_{2}+\boldsymbol{\alpha}_{2}^{*}\right) .
$$

Therefore, for any optimal solution $\boldsymbol{\alpha}_{\epsilon}^{(*)}$ of $D_{\epsilon}, \epsilon>0, \mathbf{e}^{T}\left(\boldsymbol{\alpha}_{\epsilon}+\boldsymbol{\alpha}_{\epsilon}^{*}\right) \leq v^{*}$.

Unlike the case of classification where $\mathbf{e}^{T} \boldsymbol{\alpha}_{C}$ is a well-defined function of $C$ if $\boldsymbol{\alpha}_{C}$ is an optimal solution of the C-SVC problem, here for $\epsilon$-SVR, for the same $D_{\epsilon}$, there may be different $\mathbf{e}^{T}\left(\boldsymbol{\alpha}_{\epsilon}+\boldsymbol{\alpha}_{\epsilon}^{*}\right)$. The main reason is that $\mathbf{e}^{T} \boldsymbol{\alpha}$ is the only linear term of the objective function of $C$-SVC, but for $D_{\epsilon}$, the linear term becomes $(\mathbf{y} / C)^{T}\left(\boldsymbol{\alpha}-\boldsymbol{\alpha}^{*}\right)+(\epsilon / C) \mathbf{e}^{T}\left(\boldsymbol{\alpha}+\boldsymbol{\alpha}^{*}\right)$. We will elaborate more on this in lemma 4, where we prove that $\mathbf{e}^{T}\left(\boldsymbol{\alpha}_{\epsilon}+\boldsymbol{\alpha}_{\epsilon}^{*}\right)$ can be a function of $\epsilon$ if $\mathbf{Q}$ is a positive definite matrix.

The following lemma shows the relation between $D_{v}$ and $D_{\epsilon}$. In particular, we show that for $0 \leq v<v^{*}$, any optimal solution of $D_{v}$ satisfies $\mathbf{e}^{T}\left(\boldsymbol{\alpha}+\boldsymbol{\alpha}^{*}\right)=v$.

Lemma 3. For any $D_{v}, 0 \leq v<v^{*}$, one of the following two situations must happen:

1. $D_{\nu}$ 's optimal solution set is part of the solution set of a $D_{\epsilon}, \epsilon>0$.

2. $D_{\nu}$ 's optimal solution set is the same as that of $D_{\epsilon}$, where $\epsilon>0$ is any one element in a unique open interval.

In addition, any optimal solution of $D_{\nu}$ satisfies $\mathbf{e}^{T}\left(\boldsymbol{\alpha}+\boldsymbol{\alpha}^{*}\right)=v$ and $\alpha_{i} \alpha_{i}^{*}=0$.

Proof. The Karush-Kuhn-Tucker (KKT) condition of $D_{v}$ shows that there exist $\rho \geq 0$ and $b$ such that

$$
\left[\begin{array}{cc}
\mathbf{Q} & -\mathbf{Q} \\
-\mathbf{Q} & \mathbf{Q}
\end{array}\right]\left[\begin{array}{c}
\boldsymbol{\alpha} \\
\boldsymbol{\alpha}^{*}
\end{array}\right]+\left[\begin{array}{c}
\mathbf{y} / C \\
-\mathbf{y} / C
\end{array}\right]+\frac{\rho}{C}\left[\begin{array}{l}
\mathbf{e} \\
\mathbf{e}
\end{array}\right]-b\left[\begin{array}{c}
\mathbf{e} \\
-\mathbf{e}
\end{array}\right]=\left[\begin{array}{c}
\boldsymbol{\lambda}-\boldsymbol{\xi} \\
\lambda^{*}-\boldsymbol{\xi}^{*}
\end{array}\right]
$$

If $\rho=0, \boldsymbol{\alpha}^{(*)}$ is an optimal solution of $D_{\epsilon}, \epsilon=0$. Then, $\mathbf{e}^{T}\left(\boldsymbol{\alpha}+\boldsymbol{\alpha}^{*}\right) \geq v^{*}>v$ causes a contradiction. 
Therefore, $\rho>0$, so the KKT condition implies that $\mathbf{e}^{T}\left(\boldsymbol{\alpha}+\boldsymbol{\alpha}^{*}\right)=v$. Then there are two possible situations:

Case 1: $\rho$ is unique: By assigning $\epsilon=\rho$, all optimal solutions of $D_{v}$ are KKT points of $D_{\epsilon}$. Hence, $D_{\nu}$ 's optimal solution set is part of that of a $D_{\epsilon}$. This $D_{\epsilon}$ is unique, as otherwise we can find another $\rho$ that satisfies equation 2.3.

Case 2: $\rho$ is not unique. That is, there are two $\rho_{1}<\rho_{2}$. Suppose $\rho_{1}$ and $\rho_{2}$ are the smallest and largest one satisfying the KKT. Again, the existence of $\rho_{1}$ and $\rho_{2}$ is based on the compactness of the optimal solution set. Then for any $\rho_{1}<\rho<\rho_{2}$, we consider the problem $D_{\epsilon}, \epsilon=\rho$. Define $\epsilon_{1} \equiv \rho_{1}$ and $\epsilon_{2} \equiv \rho_{2}$. From lemma 2 , since $\epsilon_{1}<\epsilon<\epsilon_{2}$,

$$
v=\mathbf{e}^{T}\left(\boldsymbol{\alpha}_{1}+\boldsymbol{\alpha}_{1}^{*}\right) \geq \mathbf{e}^{T}\left(\boldsymbol{\alpha}+\boldsymbol{\alpha}^{*}\right) \geq \mathbf{e}^{T}\left(\boldsymbol{\alpha}_{2}+\boldsymbol{\alpha}_{2}^{*}\right)=v,
$$

where $\boldsymbol{\alpha}^{(*)}$ is any optimal solution of $D_{\epsilon}$, and $\boldsymbol{\alpha}_{1}^{(*)}$ and $\boldsymbol{\alpha}_{2}^{(*)}$ are optimal solutions of $D_{\epsilon_{1}}$ and $D_{\epsilon_{2}}$, respectively. Hence, all optimal solutions of $D_{\epsilon}$ satisfy $\mathbf{e}^{T}\left(\boldsymbol{\alpha}+\boldsymbol{\alpha}^{*}\right)=v$ and all KKT conditions of $D_{\nu}$ so $D_{\epsilon}{ }^{\prime}$ s optimal solution set is in that of $D_{\nu}$.

Hence, $D_{v}$ and $D_{\epsilon}$ share at least one optimal solution $\boldsymbol{\alpha}^{(*)}$. For any other optimal solution $\overline{\boldsymbol{\alpha}}^{(*)}$ of $D_{\nu}$, it is feasible for $\left(D_{\epsilon}\right)$. Since

$$
\begin{aligned}
& \frac{1}{2}\left(\overline{\boldsymbol{\alpha}}-\overline{\boldsymbol{\alpha}}^{*}\right)^{T} \mathbf{Q}\left(\overline{\boldsymbol{\alpha}}-\overline{\boldsymbol{\alpha}}^{*}\right)+(\mathbf{y} / C)^{T}\left(\overline{\boldsymbol{\alpha}}-\overline{\boldsymbol{\alpha}}^{*}\right) \\
& \quad=\frac{1}{2}\left(\boldsymbol{\alpha}-\boldsymbol{\alpha}^{*}\right)^{T} \mathbf{Q}\left(\boldsymbol{\alpha}-\boldsymbol{\alpha}^{*}\right)+(\mathbf{y} / C)^{T}\left(\boldsymbol{\alpha}-\boldsymbol{\alpha}^{*}\right),
\end{aligned}
$$

and $\mathbf{e}^{T}\left(\overline{\boldsymbol{\alpha}}+\overline{\boldsymbol{\alpha}}^{*}\right) \leq v$, we have

$$
\begin{aligned}
& \frac{1}{2}\left(\overline{\boldsymbol{\alpha}}-\overline{\boldsymbol{\alpha}}^{*}\right)^{T} \mathbf{Q}\left(\overline{\boldsymbol{\alpha}}-\overline{\boldsymbol{\alpha}}^{*}\right)+(\mathbf{y} / C)^{T}\left(\overline{\boldsymbol{\alpha}}-\overline{\boldsymbol{\alpha}}^{*}\right)+(\epsilon / C) \mathbf{e}^{T}\left(\overline{\boldsymbol{\alpha}}+\overline{\boldsymbol{\alpha}}^{*}\right) \\
& \quad \leq \frac{1}{2}\left(\boldsymbol{\alpha}-\boldsymbol{\alpha}^{*}\right)^{T} \mathbf{Q}\left(\boldsymbol{\alpha}-\boldsymbol{\alpha}^{*}\right)+(\mathbf{y} / C)^{T}\left(\boldsymbol{\alpha}-\boldsymbol{\alpha}^{*}\right)+(\epsilon / C) \mathbf{e}^{T}\left(\boldsymbol{\alpha}+\boldsymbol{\alpha}^{*}\right)
\end{aligned}
$$

Therefore, all optimal solutions of $D_{v}$ are also optimal for $D_{\epsilon}$. Hence, $D_{\nu}$ 's optimal solution set is the same as that of $D_{\epsilon}$, where $\epsilon>0$ is any one element in a unique open interval $\left(\rho_{1}, \rho_{2}\right)$.

Finally, as $D_{\nu}$ 's optimal solution set is the same as or part of a $D_{\epsilon}$, from lemma 1 , we have $\alpha_{i} \alpha_{i}^{*}=0$.

Using the above results, we now summarize a main theorem:

Theorem 1. We have:

1. $v^{*} \leq 1$.

2. For any $v \in\left[v^{*}, 1\right], D_{\nu}$ has the same optimal objective value as $D_{\epsilon}, \epsilon=0$. 
3. For any $v \in\left[0, v^{*}\right)$, lemma 3 holds. That is, one of the following two situations must happen: (a) $D_{v}$ 's optimal solution set is part of the solution set of $D_{\epsilon}, \epsilon>0$, or (b) $D_{\nu}$ 's optimal solution set is the same as that of $D_{\epsilon}$, where $\epsilon>0$ is any one element in a unique open interval.

4. For all $D_{v}, 0 \leq v \leq 1$, there are always optimal solutions that happen at the equality $\mathbf{e}^{T}\left(\boldsymbol{\alpha}+\boldsymbol{\alpha}^{*}\right)=v$.

Proof. From the explanation after definition 1, there exists an optimal solution of $D_{\epsilon}, \epsilon=0$, which satisfies $\mathbf{e}^{T}\left(\boldsymbol{\alpha}+\boldsymbol{\alpha}^{*}\right)=v^{*}$. Then this $\boldsymbol{\alpha}^{(*)}$ must satisfy $\alpha_{i} \alpha_{i}^{*}=0, i=1, \ldots, l$, so $v^{*} \leq 1$. In addition, this $\boldsymbol{\alpha}^{(*)}$ is also feasible to $D_{v}, v \geq v^{*}$. Since $D_{v}$ has the same objective function as $D_{\epsilon}, \epsilon=0$, but has one more constraint, this solution of $D_{\epsilon}, \epsilon=0$, is also optimal for $D_{\nu}$. Hence, $D_{v}$ and $D_{v^{*}}$ have the same optimal objective value.

For $0 \leq v<v^{*}$, we already know from theorem 3 that the optimal solution happens only at the equality. For $1 \geq v \geq v^{*}$, first we know that $D_{\epsilon}, \epsilon=0$ has an optimal solution $\boldsymbol{\alpha}^{(*)}$ that satisfies $\mathbf{e}^{T}\left(\boldsymbol{\alpha}+\boldsymbol{\alpha}^{*}\right)=v^{*}$. Then we can increase some elements of $\boldsymbol{\alpha}$ and $\boldsymbol{\alpha}^{*}$ such that new vectors $\hat{\boldsymbol{\alpha}}^{(*)}$ satisfy $\mathbf{e}^{T}\left(\hat{\boldsymbol{\alpha}}+\hat{\boldsymbol{\alpha}}^{*}\right)=v$ but $\hat{\boldsymbol{\alpha}}-\hat{\boldsymbol{\alpha}}^{*}=\boldsymbol{\alpha}-\boldsymbol{\alpha}^{*}$. Hence, $\hat{\boldsymbol{\alpha}}^{(*)}$ is an optimal solution of $D_{v}$, which satisfies the equality constraint.

Therefore, the above results ensure that it is safe to solve the following problem instead of $D_{\nu}$ :

$$
\begin{gathered}
\min \frac{1}{2}\left(\boldsymbol{\alpha}-\boldsymbol{\alpha}^{*}\right)^{T} \mathbf{Q}\left(\boldsymbol{\alpha}-\boldsymbol{\alpha}^{*}\right)+(\mathbf{y} / C)^{T}\left(\boldsymbol{\alpha}-\boldsymbol{\alpha}^{*}\right) \\
\mathbf{e}^{T}\left(\boldsymbol{\alpha}-\boldsymbol{\alpha}^{*}\right)=0, \mathbf{e}^{T}\left(\boldsymbol{\alpha}+\boldsymbol{\alpha}^{*}\right)=v, \\
0 \leq \alpha_{i}, \alpha_{i}^{*} \leq 1 / l, \quad i=1, \ldots, l .
\end{gathered}
$$

This result is important because existing SVM algorithms have been able to handle these equalities easily.

Note that for $v$-SVC, there is also a $v^{*}$ where for $v \in\left(v^{*}, 1\right], D_{v}$ is infeasible. At that time, $v^{*}=2 \mathrm{~min}$ (\#positive data, \#negative data) $/ l$ can be easily calculated (Crisp \& Burges 2000). Now for $v$-SVR, it is difficult to know $v^{*}$ a priori. However, we do not have to worry about this. If a $\bar{D}_{v}, v>v^{*}$ is solved, a solution with its objective value equal to that of $D_{\epsilon}, \epsilon=0$ is obtained. Then some $\alpha_{i}$ and $\alpha_{i}^{*}$ may both be nonzero.

Since there are always optimal solutions of the dual problem that satisfy $\mathbf{e}^{T}\left(\boldsymbol{\alpha}+\boldsymbol{\alpha}^{*}\right)=v$, this also implies that the $\epsilon \geq 0$ constraint of $P_{v}$ is not necessary. In the following theorem, we derive the same result directly from the primal side:

Theorem 2. Consider a problem that is the same as $P_{v}$ but without the inequality constraint $\epsilon \geq 0$. We have that for any $0<v<1$, any optimal solution of $P_{v}$ must satisfy $\epsilon \geq 0$. 
Proof. Assume $\left(\mathbf{w}, b, \boldsymbol{\xi}, \boldsymbol{\xi}^{*}, \epsilon\right)$ is an optimal solution with $\epsilon<0$. Then for each $i$,

$$
-\epsilon-\xi_{i}^{*} \leq \mathbf{w}^{T} \phi\left(\mathbf{x}_{i}\right)+b-y_{i} \leq \epsilon+\xi_{i}
$$

implies

$$
\xi_{i}+\xi_{i}^{*}+2 \epsilon \geq 0
$$

With equation 2.4,

$$
\begin{aligned}
-0-\max \left(0, \xi_{i}^{*}+\epsilon\right) \leq-\epsilon-\xi_{i}^{*} & \leq \mathbf{w}^{T} \phi\left(\mathbf{x}_{i}\right)+b-y_{i} \\
& \leq 0+\epsilon+\xi_{i} \leq 0+\max \left(0, \xi_{i}+\epsilon\right) .
\end{aligned}
$$

Hence $\left(\mathbf{w}, b, \max (0, \boldsymbol{\xi}+\epsilon \mathbf{e}), \max \left(0, \boldsymbol{\xi}^{*}+\epsilon \mathbf{e}\right), 0\right)$ is a feasible solution of $P_{\nu}$. From equation 2.5,

$$
\max \left(0, \xi_{i}+\epsilon\right)+\max \left(0, \xi_{i}^{*}+\epsilon\right) \leq \xi_{i}+\xi_{i}^{*}+\epsilon .
$$

Therefore, with $\epsilon<0$ and $0<v<1$,

$$
\begin{aligned}
\frac{1}{2} \mathbf{w}^{T} \mathbf{w}+C\left(v \epsilon+\frac{1}{l} \sum_{i=1}^{l}\left(\xi_{i}+\xi_{i}^{*}\right)\right) \\
>\frac{1}{2} \mathbf{w}^{T} \mathbf{w}+\frac{C}{l}\left(l \epsilon+\sum_{i=1}^{l}\left(\xi_{i}+\xi_{i}^{*}\right)\right) \\
\geq \frac{1}{2} \mathbf{w}^{T} \mathbf{w}+\frac{C}{l} \sum_{i=1}^{l}\left(\max \left(0, \xi_{i}+\epsilon\right)+\max \left(0, \xi_{i}^{*}+\epsilon\right)\right)
\end{aligned}
$$

implies that $\left(\mathbf{w}, b, \boldsymbol{\xi}, \boldsymbol{\xi}^{*}\right)$ is not an optimal solution. Therefore, any optimal solution of $P_{v}$ must satisfy $\epsilon \geq 0$.

Next we demonstrate an example where one $D_{\epsilon}$ corresponds to many $D_{\nu}$. Given two training points, $\mathbf{x}_{1}=0, \mathbf{x}_{2}=0$, and target values $y_{1}=-\Delta<0$ and $y_{2}=\Delta>0$. When $\epsilon=\Delta$, if the linear kernel is used and $C=1, D_{\epsilon}$ becomes

$$
\begin{aligned}
\min & 2 \Delta\left(\alpha_{1}^{*}+\alpha_{2}\right) \\
& 0 \leq \alpha_{1}, \alpha_{1}^{*}, \alpha_{2}, \alpha_{2}^{*} \leq 1 / l, \\
& \alpha_{1}-\alpha_{1}^{*}+\alpha_{2}-\alpha_{2}^{*}=0 .
\end{aligned}
$$

Thus, $\alpha_{1}^{*}=\alpha_{2}=0$, so any $0 \leq \alpha_{1}=\alpha_{2}^{*} \leq 1 / l$ is an optimal solution. Therefore, for this $\epsilon$, the possible $\mathbf{e}^{T}\left(\boldsymbol{\alpha}+\boldsymbol{\alpha}^{*}\right)$ ranges from 0 to 1 . The relation between $v$ and $\epsilon$ is illustrated in Figure 1. 


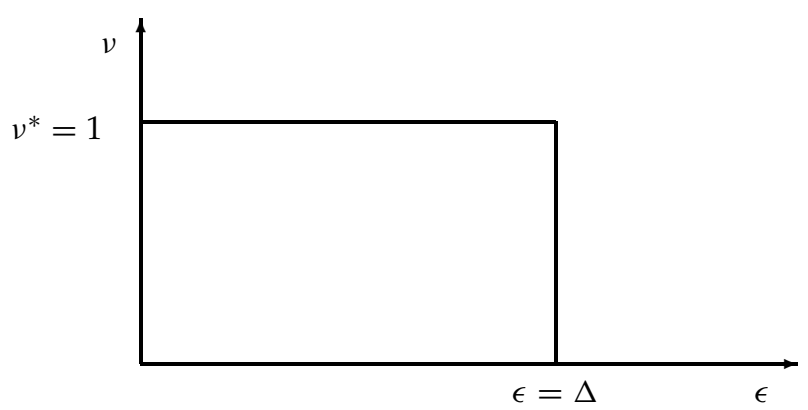

Figure 1: An example where one $D_{\epsilon}$ corresponds to different $D_{v}$

\section{When the Kernel Matrix Q Is Positive Definite}

In the previous section, we showed that for $\epsilon-S V R, \mathbf{e}^{T}\left(\boldsymbol{\alpha}_{\epsilon}+\boldsymbol{\alpha}_{\epsilon}^{*}\right)$ may not be a well-defined function of $\epsilon$, where $\boldsymbol{\alpha}_{\epsilon}^{(*)}$ is any optimal solution of $D_{\epsilon}$. Because of this difficulty, we cannot exactly apply results on the relation between C-SVC and $\nu$-SVC to $\epsilon$-SVR and $\nu$-SVR. In this section, we show that if $\mathbf{Q}$ is positive definite, then $\mathbf{e}^{T}\left(\boldsymbol{\alpha}_{\epsilon}+\boldsymbol{\alpha}_{\epsilon}^{*}\right)$ is a function of $\epsilon$, and all results discussed in Chang and Lin (2001b) hold.

Assumption 1. $\mathbf{Q}$ is positive definite.

Lemma 4. If $\epsilon>0$, then $D_{\epsilon}$ has a unique optimal solution. Therefore, we can define a function $\mathbf{e}^{T}\left(\boldsymbol{\alpha}_{\epsilon}+\boldsymbol{\alpha}_{\epsilon}^{*}\right)$ on $\epsilon$, where $\boldsymbol{\alpha}_{\epsilon}^{(*)}$ is the optimal solution of $D_{\epsilon}$.

Proof. Since $D_{\epsilon}$ is a convex problem, if $\boldsymbol{\alpha}_{1}^{(*)}$ and $\boldsymbol{\alpha}_{2}^{(*)}$ are both optimal solutions, for all $0 \leq \lambda \leq 1$,

$$
\begin{aligned}
& \frac{1}{2}\left(\lambda\left(\boldsymbol{\alpha}_{1}-\boldsymbol{\alpha}_{1}^{*}\right)+(1-\lambda)\left(\boldsymbol{\alpha}_{2}-\boldsymbol{\alpha}_{2}^{*}\right)\right)^{T} \mathbf{Q}\left(\lambda\left(\boldsymbol{\alpha}_{1}-\boldsymbol{\alpha}_{1}^{*}\right)+(1-\lambda)\left(\boldsymbol{\alpha}_{2}-\boldsymbol{\alpha}_{2}^{*}\right)\right) \\
& +(\mathbf{y} / C)^{T}\left(\lambda\left(\boldsymbol{\alpha}_{1}-\boldsymbol{\alpha}_{1}^{*}\right)+(1-\lambda)\left(\boldsymbol{\alpha}_{2}-\boldsymbol{\alpha}_{2}^{*}\right)\right) \\
& +(\epsilon / C) \mathbf{e}^{T}\left(\lambda\left(\boldsymbol{\alpha}_{1}+\boldsymbol{\alpha}_{1}^{*}\right)+(1-\lambda)\left(\boldsymbol{\alpha}_{2}+\boldsymbol{\alpha}_{2}^{*}\right)\right) \\
& =\lambda\left(\frac{1}{2}\left(\boldsymbol{\alpha}_{1}-\boldsymbol{\alpha}_{1}^{*}\right)^{T} \mathbf{Q}\left(\boldsymbol{\alpha}_{1}-\boldsymbol{\alpha}_{1}^{*}\right)\right. \\
& \left.\quad+(\mathbf{y} / C)^{T}\left(\boldsymbol{\alpha}_{1}-\boldsymbol{\alpha}_{1}^{*}\right)+(\epsilon / C) \mathbf{e}^{T}\left(\boldsymbol{\alpha}_{1}+\boldsymbol{\alpha}_{1}^{*}\right)\right) \\
& +(1-\lambda)\left(\frac{1}{2}\left(\boldsymbol{\alpha}_{2}-\boldsymbol{\alpha}_{2}^{*}\right)^{T} \mathbf{Q}\left(\boldsymbol{\alpha}_{2}-\boldsymbol{\alpha}_{2}^{*}\right)\right. \\
& \left.\quad+(\mathbf{y} / C)^{T}\left(\boldsymbol{\alpha}_{2}-\boldsymbol{\alpha}_{2}^{*}\right)+(\epsilon / C) \mathbf{e}^{T}\left(\boldsymbol{\alpha}_{2}+\boldsymbol{\alpha}_{2}^{*}\right)\right)
\end{aligned}
$$


This implies

$$
\begin{aligned}
\left(\boldsymbol{\alpha}_{1}-\boldsymbol{\alpha}_{1}^{*}\right)^{T} \mathbf{Q}\left(\boldsymbol{\alpha}_{2}-\boldsymbol{\alpha}_{2}^{*}\right)= & \frac{1}{2}\left(\boldsymbol{\alpha}_{1}-\boldsymbol{\alpha}_{1}^{*}\right)^{T} \mathbf{Q}\left(\boldsymbol{\alpha}_{1}-\boldsymbol{\alpha}_{1}^{*}\right) \\
& +\frac{1}{2}\left(\boldsymbol{\alpha}_{2}-\boldsymbol{\alpha}_{2}^{*}\right)^{T} \mathbf{Q}\left(\boldsymbol{\alpha}_{2}-\boldsymbol{\alpha}_{2}^{*}\right)
\end{aligned}
$$

Since $\mathbf{Q}$ is positive semidefinite, $\mathbf{Q}=\mathbf{L}^{T} \mathbf{L}$, so equation 3.1 implies $\| \mathbf{L}\left(\boldsymbol{\alpha}_{1}-\right.$ $\left.\boldsymbol{\alpha}_{1}^{*}\right)-\mathbf{L}\left(\boldsymbol{\alpha}_{2}-\boldsymbol{\alpha}_{2}^{*}\right) \|=0$. Hence, $\mathbf{L}\left(\boldsymbol{\alpha}_{1}-\boldsymbol{\alpha}_{1}^{*}\right)=\mathbf{L}\left(\boldsymbol{\alpha}_{2}-\boldsymbol{\alpha}_{2}^{*}\right)$. Since $\mathbf{Q}$ is positive definite, $\mathbf{L}$ is invertible, so $\boldsymbol{\alpha}_{1}-\boldsymbol{\alpha}_{1}^{*}=\boldsymbol{\alpha}_{2}-\boldsymbol{\alpha}_{2}^{*}$. Since $\epsilon>0$, from lemma 1, $\left(\alpha_{1}\right)_{i}\left(\alpha_{1}^{*}\right)_{i}=0$ and $\left(\alpha_{2}\right)_{i}\left(\alpha_{2}^{*}\right)_{i}=0$. Thus, we have $\boldsymbol{\alpha}_{1}=\boldsymbol{\alpha}_{2}$ and $\boldsymbol{\alpha}_{1}^{*}=\boldsymbol{\alpha}_{2}^{*}$.

For convex optimization problems, if the Hessian is positive definite, there is a unique optimal solution. Unfortunately, here the Hessian is $\left[\begin{array}{cc}\mathbf{Q} & -\mathbf{Q} \\ -\mathbf{Q} & \mathbf{Q}\end{array}\right]$, which is only positive semidefinite. Hence, special efforts are needed for proving the property of the unique optimal solution.

Note that in the above proof, $\mathbf{L}\left(\boldsymbol{\alpha}_{1}-\boldsymbol{\alpha}_{1}^{*}\right)=\mathbf{L}\left(\boldsymbol{\alpha}_{2}-\boldsymbol{\alpha}_{2}^{*}\right)$ implies $\left(\boldsymbol{\alpha}_{1}-\right.$ $\left.\boldsymbol{\alpha}_{1}^{*}\right)^{T} \mathbf{Q}\left(\boldsymbol{\alpha}_{1}-\boldsymbol{\alpha}_{1}^{*}\right)=\left(\boldsymbol{\alpha}_{2}-\boldsymbol{\alpha}_{2}^{*}\right)^{T} \mathbf{Q}\left(\boldsymbol{\alpha}_{2}-\boldsymbol{\alpha}_{2}^{*}\right)$. Since $\boldsymbol{\alpha}_{1}^{(*)}$ and $\boldsymbol{\alpha}_{2}^{(*)}$ are both optimal solutions, they have the same objective value so $-\mathbf{y}^{T}\left(\boldsymbol{\alpha}_{1}-\boldsymbol{\alpha}_{1}^{*}\right)+$ $\epsilon \mathbf{e}^{T}\left(\boldsymbol{\alpha}_{1}+\boldsymbol{\alpha}_{1}^{*}\right)=-\mathbf{y}^{T}\left(\boldsymbol{\alpha}_{2}-\boldsymbol{\alpha}_{2}^{*}\right)+\epsilon \mathbf{e}^{T}\left(\boldsymbol{\alpha}_{2}+\boldsymbol{\alpha}_{2}^{*}\right)$. This is not enough for proving that $\mathbf{e}^{T}\left(\boldsymbol{\alpha}_{1}+\boldsymbol{\alpha}_{1}^{*}\right)=\mathbf{e}^{T}\left(\boldsymbol{\alpha}_{2}+\boldsymbol{\alpha}_{2}^{*}\right)$. On the contrary, for $v$-SVC, the objective function is $\frac{1}{2} \boldsymbol{\alpha}^{T} \mathbf{Q} \boldsymbol{\alpha}-\mathbf{e}^{T} \boldsymbol{\alpha}$, so without the positive definite assumption, $\boldsymbol{\alpha}_{1}^{T} \mathbf{Q} \boldsymbol{\alpha}_{1}=\boldsymbol{\alpha}_{2}^{T} \mathbf{Q} \boldsymbol{\alpha}_{2}$ already implies $\mathbf{e}^{T} \boldsymbol{\alpha}_{1}=\mathbf{e}^{T} \boldsymbol{\alpha}_{2}$. Thus, $\mathbf{e}^{T} \boldsymbol{\alpha}_{C}$ is a function of $C$.

We then state some parallel results in Chang and Lin (2001b) without proofs:

Theorem 3. If $Q$ is positive definite, then the relation between $D_{v}$ and $D_{\epsilon}$ is summarized as follows:

1. (a) For any $1 \geq v \geq v^{*}, D_{\nu}$ has the same optimal objective value as $D_{\epsilon}, \epsilon=0$. (b) For any $v \in\left[0, v^{*}\right), D_{v}$ has a solution that is the same as that of either one $D_{\epsilon}, \epsilon>0$, or some $D_{\epsilon}$, where $\epsilon$ is any number in an interval.

2. If $\alpha_{\epsilon}^{*}$ is the optimal solution of $D_{\epsilon}, \epsilon>0$, the relation between $v$ and $\epsilon$ is as follows: There are $0<\epsilon_{1}<\cdots<\epsilon_{s}$ and $A_{i}, B_{i}, i=1, \ldots$, s such that

$$
\mathbf{e}^{T}\left(\boldsymbol{\alpha}_{\epsilon}+\boldsymbol{\alpha}_{\epsilon}^{*}\right)= \begin{cases}v^{*} & 0<\epsilon \leq \epsilon_{1}, \\ A_{i}+B_{i} \epsilon & \epsilon_{i} \leq \epsilon \leq \epsilon_{i+1}, i=1, \ldots, s-1, \\ 0 & \epsilon_{s} \leq \epsilon,\end{cases}
$$

where $\boldsymbol{\alpha}_{\epsilon}^{(*)}$ is the optimal solution of $D_{\epsilon}$. We also have

$$
A_{i}+B_{i} \epsilon_{i+1}=A_{i+1}+B_{i+1} \epsilon_{i+1}, i=1, \ldots, s-2,
$$


and

$$
A_{s-1}+B_{s-1} \epsilon_{s}=0 .
$$

In addition, $B_{i} \leq 0, i=1, \ldots, s-1$.

The second result of theorem 3 shows that $v$ is a piece-wise linear function of $\epsilon$. In addition, it is always decreasing.

\section{Some Issues Specific to Regression}

The motivation of $v$-SVR is that it may not be easy to decide the parameter $\epsilon$. Hence, here we are interested in the possible range of $\epsilon$. As expected, results show that $\epsilon$ is related to the target values $\mathbf{y}$.

Theorem 4. The zero vector is an optimal solution of $D_{\epsilon}$ if and only if

$$
\epsilon \geq \frac{\max _{i=1, \ldots, l} y_{i}-\min _{i=1, \ldots, l} y_{i}}{2}
$$

Proof. If the zero vector is an optimal solution of $D_{\epsilon}$, the KKT condition implies that there is a $b$ such that

$$
\left[\begin{array}{c}
\mathbf{y} \\
-\mathbf{y}
\end{array}\right]+\epsilon\left[\begin{array}{l}
\mathbf{e} \\
\mathbf{e}
\end{array}\right]-b\left[\begin{array}{c}
\mathbf{e} \\
-\mathbf{e}
\end{array}\right] \geq 0
$$

Hence, $\epsilon-b \geq-y_{i}$ and $\epsilon+b \geq y_{i}$, for all $i$. Therefore,

$$
\epsilon-b \geq-\min _{i=1, \ldots, l} y_{i} \text { and } \epsilon+b \geq \max _{i=1, \ldots, l} y_{i}
$$

so

$$
\epsilon \geq \frac{\max _{i=1, \ldots, l} y_{i}-\min _{i=1, \ldots, l} y_{i}}{2}
$$

On the other hand, if equation 4.1 is true, we can easily check that $\boldsymbol{\alpha}=$ $\boldsymbol{\alpha}^{*}=0$ satisfy the KKT condition so the zero vector is an optimal solution of $D_{\epsilon}$.

Therefore, when using $\epsilon-\mathrm{SVR}$, the largest value of $\epsilon$ to try is $\left(\max _{i=1, \ldots, l} y_{i}-\right.$ $\left.\min _{i=1, \ldots, l} y_{i}\right) / 2$.

On the other hand, $\epsilon$ should not be too small; if $\epsilon \rightarrow 0$, most data are support vectors, and overfitting tends to happen. Unfortunately, we have not been able to find an effective lower bound on $\epsilon$. However, intuitively we would think that it is also related to the target values $\mathbf{y}$. 
As the effective range of $\epsilon$ is affected by the target values $y$, a way to solve this difficulty for $\epsilon$-SVM is by scaling the target valves before training the data. For example, if all target values are scaled to $[-1,+1]$, then the effective range of $\epsilon$ will be $[0,1]$, the same as that of $\nu$. Then it may be easier to choose $\epsilon$.

There are other reasons to scale the target values. For example, we encountered some situations where if the target values $\mathbf{y}$ are not properly scaled, it is difficult to adjust the value of $C$. In particular, if $y_{i}, i=1, \ldots, l$ are large numbers and $C$ is chosen to be a small number, the approximating function is nearly a constant.

\section{Algorithms}

The algorithm considered here for $v$-SVR is similar to the decomposition method in Chang and Lin (2001b) for $v$-SVC. The implementation is part of the software LIBSVM (Chang \& Lin, 2001a). Another SVM software that has also implemented $v$-SVR is mySVM (Rüping, 2000).

The basic idea of the decomposition method is that in each iteration, the indices $\{1, \ldots, l\}$ of the training set are separated to two sets $B$ and $N$, where $B$ is the working set and $N=\{1, \ldots, l\} \backslash B$. The vector $\boldsymbol{\alpha}_{N}$ is fixed, and then a subproblem with the variable $\boldsymbol{\alpha}_{B}$ is solved.

The decomposition method was first proposed for SVM classification (Osuna, Freund, \& Girosi, 1997; Joachims, 1998; Platt, 1998). Extensions to $\epsilon-$ SVR are in, for example, Keerthi, Shevade, Bhattacharyya, \& Murthy (2000) and Laskov (2002). The main difference of these methods is their working set selections, which may significantly affect the number of iterations. Due to the additional equality 1.3 in the $v$-SVM, more considerations on the working set selection are needed. (Discussions on classification are in Keerthi \& Gilbert, 2002, and Chang \& Lin, 2001b.)

For consistency with other SVM formulations in LIBSVM, we consider $D_{v}$ as the following scaled form:

$$
\begin{aligned}
\min _{\bar{\alpha}} & \frac{1}{2} \bar{\alpha}^{T} \overline{\mathbf{Q}} \bar{\alpha}+\overline{\mathbf{p}}^{T} \bar{\alpha} \\
& \overline{\mathbf{y}}^{T} \bar{\alpha}=\Delta_{1}, \\
& \overline{\mathbf{e}}^{T} \bar{\alpha}=\Delta_{2}, \\
& 0 \leq \bar{\alpha}_{t} \leq C, t=1, \ldots, 2 l,
\end{aligned}
$$

where

$$
\begin{aligned}
\overline{\mathbf{Q}}= & {\left[\begin{array}{cc}
\mathbf{Q} & -\mathbf{Q} \\
-\mathbf{Q} & \mathbf{Q}
\end{array}\right], \bar{\alpha}=\left[\begin{array}{c}
\boldsymbol{\alpha} \\
\boldsymbol{\alpha}^{*}
\end{array}\right], \overline{\mathbf{p}}=\left[\begin{array}{l}
\mathbf{y} \\
\mathbf{y}
\end{array}\right], \overline{\mathbf{y}}=\left[\begin{array}{c}
\mathbf{e} \\
-\mathbf{e}
\end{array}\right], \overline{\mathbf{e}}=\left[\begin{array}{l}
\mathbf{e} \\
\mathbf{e}
\end{array}\right], } \\
& \Delta_{1}=0, \Delta_{2}=C l \nu .
\end{aligned}
$$


That is, we replace $C / l$ by $C$. Note that because of the result in theorem 1 , we are safe to use an equality constraint here in equation 5.1.

Then the subproblem is as follows:

$$
\begin{aligned}
\min _{\bar{\alpha}_{B}} & \frac{1}{2} \bar{\alpha}_{B}^{T} \overline{\mathbf{Q}}_{B B} \bar{\alpha}_{B}+\left(\overline{\mathbf{p}}_{B}+\overline{\mathbf{Q}}_{B N} \bar{\alpha}_{N}^{k}\right)^{T} \bar{\alpha}_{B} \\
& \overline{\mathbf{y}}_{B}^{T} \bar{\alpha}_{B}=\Delta_{1}-\overline{\mathbf{y}}_{N}^{T} \bar{\alpha}_{N}, \\
& \overline{\mathbf{e}}_{B}^{T} \bar{\alpha}_{B}=\Delta_{2}-\overline{\mathbf{e}}_{N}^{T} \bar{\alpha}_{N}, \\
& 0 \leq\left(\bar{\alpha}_{B}\right)_{t} \leq C, t=1, \ldots, q,
\end{aligned}
$$

where $q$ is the size of the working set.

Following the idea of sequential minimal optimization (SMO) by Platt (1998), we use only two elements as the working set in each iteration. The main advantage is that an analytic solution of equation 5.2 can be obtained so there is no need to use an optimization software.

Our working set selection follows from Chang and lin (2001b), which is a modification of the selection in the software SVM light (Joachims, 1998). Since they dealt with the case of more general selections where the size is not restricted to two, here we have a simpler derivation directly using the KKT condition. It is similar to that in Keerthi and Gilbert (2002, section 5).

Now if only two elements $i$ and $j$ are selected but $\bar{y}_{i} \neq \bar{y}_{j}$, then $\overline{\mathbf{y}}_{B}^{T} \bar{\alpha}_{B}=$ $\Delta_{1}-\overline{\mathbf{y}}_{N}^{T} \bar{\alpha}_{N}$ and $\overline{\mathbf{e}}_{B}^{T} \bar{\alpha}_{B}=\Delta_{2}-\overline{\mathbf{e}}_{N}^{T} \bar{\alpha}_{N}$ imply that there are two equations with two variables, so in general equation 5.2 has only one feasible point. Therefore, from $\bar{\alpha}_{k}$, the solution of the $k$ th iteration, it cannot be moved any more. On the other hand, if $\bar{y}_{i}=\bar{y}_{j}, \overline{\mathbf{y}}_{B}^{T} \bar{\alpha}_{B}=\Delta_{1}-\overline{\mathbf{y}}_{N}^{T} \bar{\alpha}_{N}$ and $\overline{\mathbf{e}}_{B}^{T} \bar{\alpha}_{B}=\Delta_{2}-\overline{\mathbf{e}}_{N}^{T} \bar{\alpha}_{N}$ become the same equality, so there are multiple feasible solutions. Therefore, we have to keep $\bar{y}_{i}=\bar{y}_{j}$ while selecting the working set.

The KKT condition of equation 5.1 shows that there are $\rho$ and $b$ such that

$$
\begin{aligned}
\nabla f(\bar{\alpha})_{i}-\rho+b \bar{y}_{i} & =0 \text { if } 0<\bar{\alpha}_{i}<C, \\
& \geq 0 \text { if } \bar{\alpha}_{i}=0, \\
& \leq 0 \text { if } \bar{\alpha}_{i}=C .
\end{aligned}
$$

Define

$$
r_{1} \equiv \rho-b, r_{2} \equiv \rho+b .
$$

If $\bar{y}_{i}=1$, the KKT condition becomes

$$
\begin{aligned}
\nabla f(\bar{\alpha})_{i}-r_{1} & \geq 0 \text { if } \bar{\alpha}_{i}<C, \\
& \leq 0 \text { if } \bar{\alpha}_{i}>0 .
\end{aligned}
$$


On the other hand, if $\bar{y}_{i}=-1$, it is

$$
\begin{aligned}
\nabla f(\bar{\alpha})_{i}-r_{2} & \geq 0 \text { if } \bar{\alpha}_{i}<C, \\
& \leq 0 \text { if } \bar{\alpha}_{i}>0
\end{aligned}
$$

Hence, indices $i$ and $j$ are selected from either

$$
\begin{aligned}
& i=\operatorname{argmin}_{t}\left\{\nabla f(\bar{\alpha})_{t} \mid \bar{y}_{t}=1, \bar{\alpha}_{t}<C\right\}, \\
& j=\operatorname{argmax}_{t}\left\{\nabla f(\bar{\alpha})_{t} \mid \bar{y}_{t}=1, \bar{\alpha}_{t}>0\right\},
\end{aligned}
$$

or

$$
\begin{aligned}
& i=\operatorname{argmin}_{t}\left\{\nabla f(\bar{\alpha})_{t} \mid \bar{y}_{t}=-1, \bar{\alpha}_{t}<C\right\}, \\
& j=\operatorname{argmax}_{t}\left\{\nabla f(\bar{\alpha})_{t} \mid \bar{y}_{t}=-1, \bar{\alpha}_{t}>0\right\},
\end{aligned}
$$

depending on which one gives a larger $\nabla f(\bar{\alpha})_{j}-\nabla f(\bar{\alpha})_{i}$ (i.e., larger KKT violations). If the selected $\nabla f(\bar{\alpha})_{j}-\nabla f(\bar{\alpha})_{i}$ is smaller than a given $\epsilon\left(10^{-3}\right.$ in our experiments), the algorithm stops.

Similar to the case of $v$-SVC, here the zero vector cannot be the initial solution. This is due to the additional equality constraint $\overline{\mathbf{e}}^{T} \bar{\alpha}=\Delta_{2}$ of equation 5.1. Here we assign both initial $\bar{\alpha}$ and $\bar{\alpha}^{*}$ with the same values. The first $\lceil\nu l / 2\rceil$ elements are $[C, \ldots, C, C(\nu l / 2-\lfloor v l / 2\rfloor)]^{T}$ while others are zero.

It has been proved that if the decomposition method of LIBSVM is used for solving $D_{\epsilon}, \epsilon>0$, during iterations $\alpha_{i} \alpha_{i}^{*}=0$ always holds (Lin, 2001, theorem 4.1). Now for $\nu$-SVR we do not have this property as $\alpha_{i}$ and $\alpha_{i}^{*}$ may both be nonzero during iterations.

Next, we discuss how to find $v^{*}$. We claim that if $\mathbf{Q}$ is positive definite and $\left(\boldsymbol{\alpha}, \boldsymbol{\alpha}^{*}\right)$ is any optimal solution of $D_{\epsilon}, \epsilon=0$, then

$$
v^{*}=\sum_{i=1}^{l}\left|\alpha_{i}-\alpha_{i}^{*}\right| \text {. }
$$

Note that by defining $\beta \equiv \alpha-\alpha^{*}, D_{\epsilon}, \epsilon=0$ is equivalent to

$$
\begin{aligned}
\min & \frac{1}{2} \boldsymbol{\beta}^{T} \mathbf{Q} \boldsymbol{\beta}+(\mathbf{y} / C)^{T} \boldsymbol{\beta} \\
& \mathbf{e}^{T} \boldsymbol{\beta}=0, \\
& -1 / l \leq \beta_{i} \leq 1 / l, \quad i=1, \ldots, l .
\end{aligned}
$$

When $Q$ is positive definite, it becomes a strictly convex programming problem, so there is a unique optimal solution $\boldsymbol{\beta}$. That is, we have a unique $\boldsymbol{\alpha}-\boldsymbol{\alpha}^{*}$ but may have multiple optimal $\left(\boldsymbol{\alpha}, \boldsymbol{\alpha}^{*}\right)$. With conditions $0 \leq \alpha_{i}, \alpha_{i}^{*} \leq 1 / l$, the calculation of $\left|\alpha_{i}-\alpha_{i}^{*}\right|$ is similar to $\alpha_{i}$ and $\alpha_{i}^{*}$ until one becomes zero. Then $\left|\alpha_{i}-\alpha_{i}^{*}\right|$ is the smallest possible $\alpha_{i}+\alpha_{i}^{*}$ with a fixed $\alpha_{i}-\alpha_{i}^{*}$. In the next section, we will use the RBF kernel, so if no data points are the same, $\mathbf{Q}$ is positive definite. 


\section{Experiments}

In this section we demonstrate some numerical comparisons between $v$ SVR and $\epsilon$-SVR. We test the RBF kernel with $Q_{i j}=e^{-\left\|\mathbf{x}_{i}-\mathbf{x}_{j}\right\|^{2} / n}$, where $n$ is the number of attributes of a training data.

The computational experiments for this section were done on a Pentium III-500 with 256 MB RAM using the gcc compiler. Our implementation is part of the software LIBSVM, which includes both $\nu$-SVR and $\epsilon$-SVR using the decomposition method. We used $100 \mathrm{MB}$ as the cache size of LIBSVM for storing recently used $Q_{i j}$. The shrinking heuristics in LIBSVM is turned off for an easier comparison.

We test problems from various collections. Problems housing, abalone, mpg, pyrimidines, and triazines are from the Statlog collection (Michie, Spiegelhetter, \& Taylor, 1994). From StatLib (http://lib.stat.cmu.edu/ datasets), we select bodyfat, space_ga, and cadata. Problem cpusmall is from the Delve archive which collects data for evaluating learning in valid experiments (http:/ / www.cs.toronto.edu/ delve). Problem mg is a MackeyGlass time series where we use the same settings as the experiments in Flake and Lawrence (2002). Thus, we predict 85 time steps in the future with six inputs. For these problems, some data entries have missing attributes, so we remove them before conducting experiments. Both the target and attribute values of these problems are scaled to $[-1,+1]$. Hence, the effective range of $\epsilon$ is $[0,1]$.

For each problem, we solve its $D_{v}$ form using $v=0.2,0.4,0.6$, and 0.8 first. Then we solve $D_{\epsilon}$ with $\epsilon=\rho$ for comparison. Tables 1 and 2 present the number of training data $(l)$, the number of iterations, and the training time by using $C=1$ and $C=100$, respectively. In the last column, we also list the number $v^{*}$ of each problem.

From both tables, we have the following observations:

1. Following theoretical results, we see that as $v$ increases, its corresponding $\epsilon$ decreases.

2. If $v \leq v^{*}$, as $v$ increases, the number of iterations of $v$-SVR and its corresponding $\epsilon-\mathrm{SVR}$ is increasing. Note that the case of $v \leq v^{*}$ covers all results in Table 1 and most of Table 2. Our explanation is as follows: When $v$ is larger, there are more support vectors, so during iterations, the number of nonzero variables is also larger. Hsu and Lin (2002) pointed out that if during iterations there are more nonzero variables than those at the optimum, the decomposition method will take many iterations to reach the final face. Here, a face means the subspace by considering only free variables. An example is in Figure 2 where we plot the number of free variables during iterations against the number of iterations. To be more precise, the $y$-axis is the number of $0<\alpha_{i}<C$ and $0<\alpha_{i}^{*}<C$ where $\left(\boldsymbol{\alpha}, \boldsymbol{\alpha}^{*}\right)$ is the solution at one iteration. We can see that for solving $\epsilon-\mathrm{SVR}$ or $\nu$-SVR, it takes a lot of iteration to identify the optimal face. From the aspect of $\epsilon-S V R$, we can consider $\epsilon \mathbf{e}^{T}\left(\boldsymbol{\alpha}+\boldsymbol{\alpha}^{*}\right)$ as a penalty term in the objective function of $D_{\epsilon}$. 
Table 1: Solving $\nu$-SVR and $\epsilon$-SVR: $C=1$ (time in seconds).

\begin{tabular}{|c|c|c|c|c|c|c|c|c|}
\hline Problem & $l$ & $v$ & $\epsilon$ & $v$ Iterations & $\epsilon$ Iterations & $v$ Time & $\epsilon$ Time & $v^{*}$ \\
\hline \multirow[t]{4}{*}{ pyrimidines } & 74 & 0.2 & 0.135131 & 181 & 145 & 0.03 & 0.02 & 0.817868 \\
\hline & & 0.4 & 0.064666 & 175 & 156 & 0.03 & 0.04 & \\
\hline & & 0.6 & 0.028517 & 365 & 331 & 0.04 & 0.03 & \\
\hline & & 0.8 & 0.002164 & 695 & 460 & 0.05 & 0.05 & \\
\hline \multirow[t]{4}{*}{ mpg } & 392 & 0.2 & 0.152014 & 988 & 862 & 0.19 & 0.16 & 0.961858 \\
\hline & & 0.4 & 0.090124 & 1753 & 1444 & 0.32 & 0.27 & \\
\hline & & 0.6 & 0.048543 & 2115 & 1847 & 0.40 & 0.34 & \\
\hline & & 0.8 & 0.020783 & 3046 & 2595 & 0.56 & 0.51 & \\
\hline \multirow[t]{4}{*}{ bodyfat } & 252 & 0.2 & 0.012700 & 1112 & 1047 & 0.14 & 0.13 & 0.899957 \\
\hline & & 0.4 & 0.006332 & 2318 & 2117 & 0.25 & 0.23 & \\
\hline & & 0.6 & 0.002898 & 3553 & 2857 & 0.37 & 0.31 & \\
\hline & & 0.8 & 0.001088 & 4966 & 3819 & 0.48 & 0.42 & \\
\hline \multirow[t]{4}{*}{ housing } & 506 & 0.2 & 0.161529 & 799 & 1231 & 0.30 & 0.34 & 0.946593 \\
\hline & & 0.4 & 0.089703 & 1693 & 1650 & 0.53 & 0.45 & \\
\hline & & 0.6 & 0.046269 & 1759 & 2002 & 0.63 & 0.60 & \\
\hline & & 0.8 & 0.018860 & 2700 & 2082 & 0.85 & 0.65 & \\
\hline \multirow[t]{4}{*}{ triazines } & 186 & 0.2 & 0.380308 & 175 & 116 & 0.13 & 0.10 & 0.900243 \\
\hline & & 0.4 & 0.194967 & 483 & 325 & 0.18 & 0.15 & \\
\hline & & 0.6 & 0.096720 & 422 & 427 & 0.20 & 0.18 & \\
\hline & & 0.8 & 0.033753 & 532 & 513 & 0.23 & 0.23 & \\
\hline \multirow[t]{4}{*}{$\mathrm{mg}$} & 1385 & 0.2 & 0.366606 & 1928 & 1542 & 1.58 & 1.18 & 0.992017 \\
\hline & & 0.4 & 0.216329 & 3268 & 3294 & 2.75 & 2.35 & \\
\hline & & 0.6 & 0.124792 & 3400 & 3300 & 3.36 & 2.76 & \\
\hline & & 0.8 & 0.059115 & 4516 & 4296 & 4.24 & 3.65 & \\
\hline \multirow[t]{4}{*}{ abalone } & 4177 & 0.2 & 0.168812 & 4189 & 3713 & 15.68 & 11.69 & 0.994775 \\
\hline & & 0.4 & 0.094959 & 8257 & 7113 & 30.38 & 22.88 & \\
\hline & & 0.6 & 0.055966 & 12,483 & 12,984 & 42.74 & 37.41 & \\
\hline & & 0.8 & 0.026165 & 18,302 & 18,277 & 65.98 & 54.04 & \\
\hline \multirow[t]{4}{*}{ space_ga } & 3107 & 0.2 & 0.087070 & 5020 & 4403 & 10.47 & 7.56 & 0.990468 \\
\hline & & 0.4 & 0.053287 & 8969 & 7731 & 18.70 & 14.44 & \\
\hline & & 0.6 & 0.032080 & 12,261 & 10,704 & 26.27 & 20.72 & \\
\hline & & 0.8 & 0.014410 & 16,311 & 13,852 & 32.71 & 27.19 & \\
\hline \multirow[t]{4}{*}{ cpusmall } & 8192 & 0.2 & 0.086285 & 8028 & 7422 & 82.66 & 59.14 & 0.990877 \\
\hline & & 0.4 & 0.054095 & 16,585 & 15,240 & 203.20 & 120.48 & \\
\hline & & 0.6 & 0.031285 & 22,376 & 19,126 & 283.71 & 163.96 & \\
\hline & & 0.8 & 0.013842 & 28,262 & 24,840 & 355.59 & 213.25 & \\
\hline \multirow[t]{4}{*}{ cadata } & 20,640 & 0.2 & 0.294803 & 12,153 & 10,961 & 575.11 & 294.53 & 0.997099 \\
\hline & & 0.4 & 0.168370 & 24,614 & 20,968 & 1096.87 & 574.77 & \\
\hline & & 0.6 & 0.097434 & 35,161 & 30,477 & 1530.01 & 851.91 & \\
\hline & & 0.8 & 0.044636 & 42,709 & 40,652 & 1883.35 & 1142.27 & \\
\hline
\end{tabular}

Hence, when $\epsilon$ is larger, fewer $\alpha_{i}, \alpha_{i}^{*}$ are nonzero. That is, the number of support vectors is fewer.

3. There are few problems (e.g., pyrimidines, bodyfat, and triazines) where $v \geq v^{*}$ is encountered. When this happens, their $\epsilon$ should be zero, but due to numerical inaccuracy, the output $\epsilon$ are only small positive numbers. Then for different $v \geq v^{*}$, when solving their corresponding $D_{\epsilon}$, the number of 
Table 2: Solving $\nu$-SVR and $\epsilon$-SVR: $C=100$ (time in seconds).

\begin{tabular}{|c|c|c|c|c|c|c|c|c|}
\hline Problem & $l$ & $v$ & $\epsilon$ & $v$ Iterations & $\epsilon$ Iterations & $v$ Time & $\epsilon$ Time & $v^{*}$ \\
\hline \multirow[t]{4}{*}{ pyrimidines } & 74 & $0.2^{*}$ & 0.000554 & 29,758 & 11,978 & 0.63 & 0.27 & 0.191361 \\
\hline & & $0.4^{*}$ & 0.000317 & 30,772 & 11,724 & 0.65 & 0.27 & \\
\hline & & $0.6^{*}$ & 0.000240 & 27,270 & 11,802 & 0.58 & 0.27 & \\
\hline & & $0.8^{*}$ & 0.000146 & 20,251 & 12,014 & 0.44 & 0.28 & \\
\hline \multirow[t]{4}{*}{ mpg } & 392 & 0.2 & 0.121366 & 85,120 & 74,878 & 9.53 & 8.26 & 0.876646 \\
\hline & & 0.4 & 0.069775 & 210,710 & 167,719 & 24.50 & 19.32 & \\
\hline & & 0.6 & 0.032716 & 347,777 & 292,426 & 42.08 & 34.82 & \\
\hline & & 0.8 & 0.007953 & 383,164 & 332,725 & 47.61 & 40.86 & \\
\hline \multirow[t]{4}{*}{ bodyfat } & 252 & 0.2 & 0.001848 & 238,927 & 164,218 & 16.80 & 11.58 & 0.368736 \\
\hline & & $0.4^{*}$ & 0.000486 & 711,157 & 323,016 & 50.77 & 23.24 & \\
\hline & & $0.6^{*}$ & 0.000291 & 644,602 & 339,569 & 46.23 & 24.33 & \\
\hline & & $0.8^{*}$ & 0.000131 & 517,370 & 356,316 & 37.28 & 25.55 & \\
\hline \multirow[t]{4}{*}{ housing } & 506 & 0.2 & 0.092998 & 154,565 & 108,220 & 24.21 & 16.87 & 0.815085 \\
\hline & & 0.4 & 0.051726 & 186,136 & 182,889 & 30.49 & 29.51 & \\
\hline & & 0.6 & 0.026340 & 285,354 & 271,278 & 48.62 & 45.64 & \\
\hline & & 0.8 & 0.002161 & 397,115 & 284,253 & 69.16 & 49.12 & \\
\hline \multirow[t]{4}{*}{ triazines } & 186 & 0.2 & 0.193718 & 16,607 & 22,651 & 0.94 & 1.20 & 0.582147 \\
\hline & & 0.4 & 0.074474 & 34,034 & 47,205 & 1.89 & 2.52 & \\
\hline & & $0.6^{*}$ & 0.000381 & 106,621 & 51,175 & 5.69 & 2.84 & \\
\hline & & $0.8^{*}$ & 0.000139 & 68,553 & 50,786 & 3.73 & 2.81 & \\
\hline \multirow[t]{4}{*}{$\mathrm{mg}$} & 1385 & 0.2 & 0.325659 & 190,065 & 195,519 & 87.99 & 89.20 & 0.966793 \\
\hline & & 0.4 & 0.189377 & 291,315 & 299,541 & 139.10 & 141.73 & \\
\hline & & 0.6 & 0.107324 & 397,449 & 407,159 & 194.81 & 196.14 & \\
\hline & & 0.8 & 0.043439 & 486,656 & 543,520 & 241.20 & 265.27 & \\
\hline \multirow[t]{4}{*}{ abalone } & 4177 & 0.2 & 0.162593 & 465,922 & 343,594 & 797.48 & 588.92 & 0.988298 \\
\hline & & 0.4 & 0.091815 & 901,275 & 829,951 & 1577.83 & 1449.37 & \\
\hline & & 0.6 & 0.053244 & $1,212,669$ & $1,356,556$ & 2193.97 & 2506.52 & \\
\hline & & 0.8 & 0.024670 & $1,680,704$ & $1,632,597$ & 2970.98 & 2987.30 & \\
\hline \multirow[t]{4}{*}{ space_ga } & 3107 & 0.2 & 0.078294 & 510,035 & 444,455 & 595.42 & 508.41 & 0.984568 \\
\hline & & 0.4 & 0.048643 & 846,873 & 738,805 & 1011.82 & 867.32 & \\
\hline & & 0.6 & 0.028933 & $1,097,732$ & $1,054,464$ & 1362.67 & 1268.40 & \\
\hline & & 0.8 & 0.013855 & $1,374,987$ & $1,393,044$ & 1778.38 & 1751.39 & \\
\hline \multirow[t]{4}{*}{ cpusmall } & 8192 & 0.2 & 0.070568 & 977,374 & 863,579 & 4304.42 & 3606.35 & 0.978351 \\
\hline & & 0.4 & 0.041640 & 1,783,725 & $1,652,396$ & 8291.12 & 7014.32 & \\
\hline & & 0.6 & 0.022280 & $2,553,150$ & $2,363,251$ & $11,673.62$ & $10,691.95$ & \\
\hline & & 0.8 & 0.009616 & $3,085,005$ & $2,912,838$ & $14,784.05$ & $12,737.35$ & \\
\hline \multirow[t]{4}{*}{ cadata } & 20640 & 0.2 & 0.263428 & $1,085,719$ & $1,081,038$ & $16,003.55$ & $15,475.36$ & 0.995602 \\
\hline & & 0.4 & 0.151341 & $2,135,097$ & $2,167,643$ & $31,936.05$ & $31,474.21$ & \\
\hline & & 0.6 & 0.087921 & $2,813,070$ & $2,614,179$ & $42,983.89$ & $38,580.61$ & \\
\hline & & 0.8 & 0.039595 & $3,599,953$ & $3,379,580$ & $54,917.10$ & $49,754.27$ & \\
\hline
\end{tabular}

Note: * Experiments where $v \geq v^{*}$.

iterations is about the same, as we essentially solve the same problem: $D_{\epsilon}$ with $\epsilon=0$.

On the other hand, surprisingly, we see that at this time as $v$ increases, it is easier to solve $D_{v}$ with fewer iterations. Now, its solution is optimal for $D_{\epsilon}, \epsilon=0$, but the larger $v$ is, the more $\left(\alpha_{i}, \alpha_{i}^{*}\right)$ are both nonzeros. Therefore, 

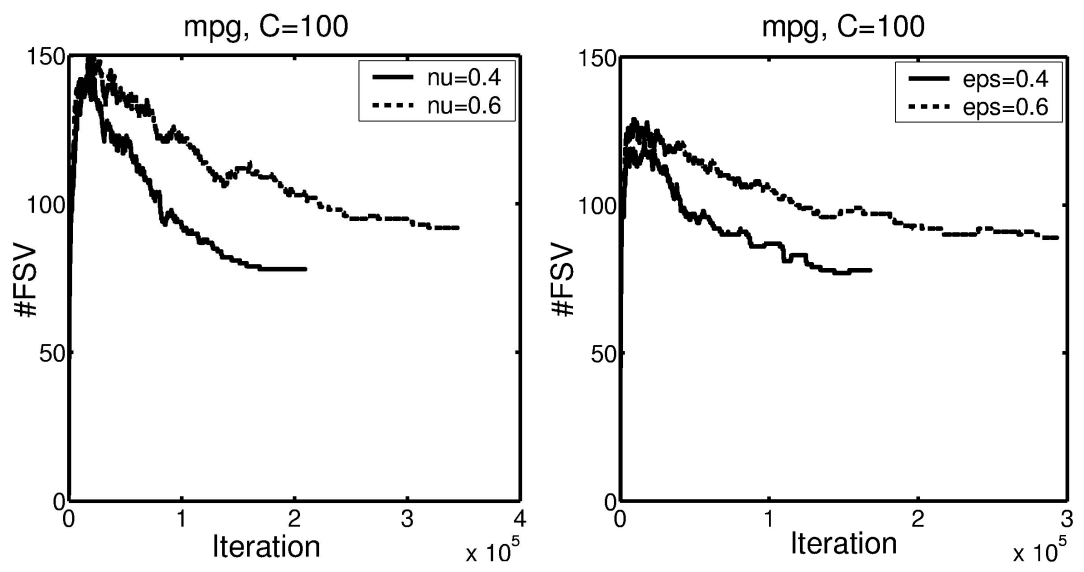

Figure 2: Iterations and number of free variables $\left(v \leq v^{*}\right)$.

contrary to the general case $v \leq v^{*}$ where it is difficult to identify and move free variables during iterations back to bounds at the optimum, there is no strong need to do so. To be more precise, in the beginning of the decomposition method, many variables become nonzero as we try to modify them for minimizing the objective function. If, finally, most of these variables are still nonzero, we do not need the efforts to put them back to bounds. In Figure 3, we plot the number of free variables against the number of iterations using problem triazines with $v=0.6,0.8$, and $\epsilon=0.000139 \approx 0$.

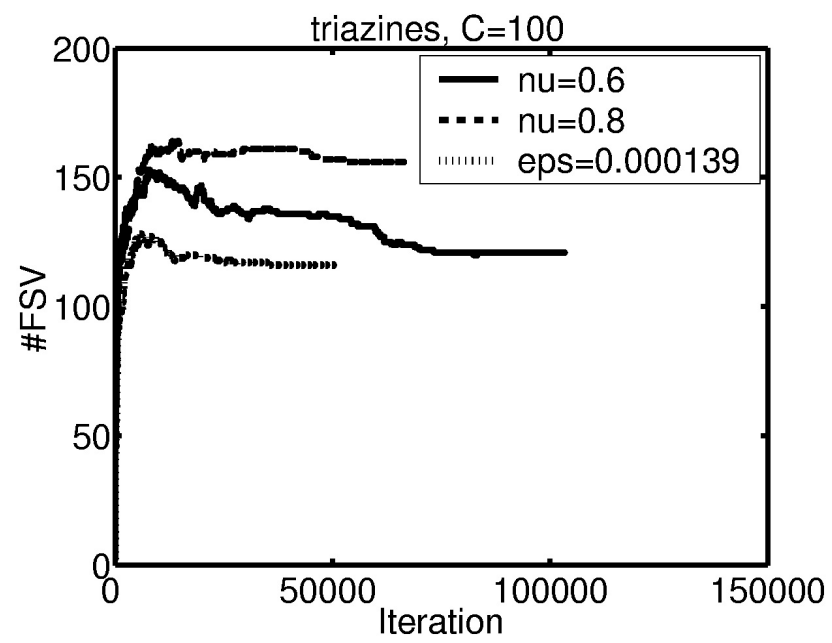

Figure 3: Iterations and number of free variables $\left(v \geq v^{*}\right)$. 
It can be clearly seen that for large $v$, the decomposition method identifies the optimal face more quickly, so the total number of iterations is fewer.

4. When $v \leq v^{*}$, we observe that there are minor differences in the number of iterations for $\epsilon$-SVR and $\nu$-SVR. In Table 1, for nearly all problems, $\nu$-SVR takes a few more iterations than $\epsilon$-SVR. However, in Table 2, for problems triazines and $\mathrm{mg}, v$-SVR is slightly faster. Note that there are several dissimilarities between algorithms for $\nu$-SVR and $\epsilon$-SVR. For example, $\epsilon$-SVR generally starts from the zero vector, but $v$-SVR has to use a nonzero initial solution. For the working set selection, the two indices selected for $\epsilon$-SVR can be any $\alpha_{i}$ or $\alpha_{i}^{*}$, but the two equality constraints lead to the selection 5.5 and 5.6 for $v$-SVR where the set is from either $\left\{\alpha_{1}, \ldots, \alpha_{l}\right\}$ or $\left\{\alpha_{1}^{*}, \ldots, \alpha_{l}^{*}\right\}$. Furthermore, as the stopping tolerance $10^{-3}$ might be too loose in some cases, the $\epsilon$ obtained after solving $D_{v}$ may be a little different from the theoretical value. Hence, we actually solve two problems with slightly different optimal solution sets. All of these factors may contribute to the distinction on iterations.

5. We see that it is much harder to solve problems using $C=100$ than using $C=1$. The difference is even more dramatic than the case of classification. We do not have a good explanation for this observation.

\section{Conclusion}

We have shown that the inequality in the $v$-SVR formulation can be treated as an equality. Hence, algorithms similar to those for $v$-SVC can be applied for $v$-SVR. In addition, in section 6, we showed similarities and dissimilarities on numerical properties of $\epsilon$-SVR and $\nu$-SVR. We think that in the future, the relation between $C$ and $v$ (or $C$ and $\epsilon$ ) should be investigated in more detail. The model selection on these parameters is also an important issue.

\section{References}

Chang, C.-C., \& Lin, C.-J. (2001a). LIBSVM: A library for support vector machines [Computer Software]. Available on-line: http://www.csie.ntu.edu.tw/ cjlin /libsvm.

Chang, C.-C., \& Lin, C.-J., (2001b). Training $v$-support vector classifiers: Theory and algorithms. Neural Computation, 13(9), 2119-2147.

Crisp, D. J., \& Burges, C. J. C. (2000). A geometric interpretation of v-SVM classifiers. In S. Solla, T. Leen, \& K.-R. Müller (Eds.), Advances in neural information processing systems, 12. Cambridge, MA: MIT Press.

Flake, G. W., \& Lawrence, S. (2002). Efficient SVM regression training with SMO. Machine Learning, 46, 271-290.

Hsu, C.-W., \& Lin, C.-J. (2002). A simple decomposition method for support vector machines. Machine Learning, 46, 291-314.

Joachims, T. (1998). Making large-scale SVM learning practical. In B. Schölkopf, C. J. C. Burges, \& A. J. Smola (Eds.), Advances in kernel methods-Support vector learning, Cambridge, MA: MIT Press. 
Keerthi, S. S., \& Gilbert, E. G. (2002). Convergence of a generalized SMO algorithm for SVM classifier design. Machine Learning, 46, 351-360.

Keerthi, S. S., Shevade, S., Bhattacharyya, C., and Murthy, K. (2000). Improvements to SMO algorithm for SVM regression. IEEE Transactions on Neural Networks, 11(5), 1188-1193.

Laskov, P. (2002). An improved decomposition algorithm for regression support vector machines. Machine Learning, 46, 315-350.

Lin, C.-J. (2001). On the convergence of the decomposition method for support vector machines. IEEE Transactions on Neural Networks, 12, 1288-1298.

Michie, D., Spiegelhalter, D. J., \& Taylor, C. C. (1994). Machine learning, neural and statistical classification. Englewood Cliffs, NJ: Prentice Hall. Available on-line at anonymous ftp: ftp.ncc.up.pt/pub/statlog/.

Osuna, E., Freund, R., \& Girosi, E. (1997). Training support vector machines: An application to face detection. In Proceedings of CVPR'97. New York: IEEE.

Platt, J. C. (1998). Fast training of support vector machines using sequential minimal optimization. In B. Schölkopf, C. J. C. Burges, \& A. J. Smola (Eds.), Advances in kernel methods-Support vector learning. Cambridge, MA: MIT Press.

Rüping, S. (2000). mySVM-another one of those support vector machines. [Computer software]. Available on-line: http://www-ai.cs.unidortmund.de/SOFTWARE/MYSVM/.

Schölkopf, B., Smola, A. J., \& Williamson, R. (1999). Shrinking the tube: A new support vector regression algorithm. In M. S. Kearns, S. A. Solla, \& D. A. Cohn (Eds.), Advances in neural information processing systems, 11, Cambridge, MA: MIT Press.

Schölkopf, B., Smola, A., Williamson, R. C., \& Bartlett, P. L. (2000). New support vector algorithms. Neural Computation, 12, 1207-1245.

Vapnik, V. (1998). Statistical learning theory. New York: Wiley.

Received April 6, 2001; accepted January 7, 2002. 